\title{
Loss decomposition in non-oriented steel sheets: the role of the classical losses.
}

\author{
Carlo S. Ragusa1, Carlo Appino2, Mahmood Khan1, Olivier de la Barrière3, Hanyu Zhao4, and Fausto Fiorillo2
}

Abstract - The concept of loss separation based on the Statistical Theory of Losses (STL) provides complete and accurate description of the frequency dependence of the energy losses in non-oriented soft magnetic sheets under the assumption of uniform magnetization reversal through the sheet cross-section. This assumption, implying a simple standard formulation for the classical loss component, has been recently challenged in the literature, in favor of a non-uniform reversal mechanism, expected to prevail in highly non-linear materials, where saturation magnetization wavefronts are deemed to symmetrically propagate across the sheet thickness (Saturation Wave Model, SWM). Different conclusions regarding the dynamic loss analysis and its decomposition into the classical and excess loss components correspondingly emerge. In this paper we discuss detailed investigations on the broadband energy loss versus frequency behavior in different non-oriented Fe-Si and low-carbon steel sheets. The experiments can be fully and consistently described by the STL. This occurs, in particular, for high-induction values and near-squared hysteresis loops, a predictable condition for adopting the SWM, which fails instead to account for the experiments.

Index Terms—Soft Magnetic Materials, Magnetic Losses, Non-oriented Steel Sheets, Classical Losses.

\section{INTRODUCTION}

The energy losses and their frequency dependence in magnetic sheets have found definite theoretical assessment and easy practical implementation with the Statistical Theory of Losses (STL). The concept of loss separation, where the total loss at a given frequency $f$ is expressed as $W(f)=W_{\mathrm{h}}+W_{\mathrm{cl}}(f)+W_{\mathrm{exc}}(f)$, the sum of hysteresis $W_{\mathrm{h}}$, classical $W_{\mathrm{cl}}(f)$, and excess $W_{\mathrm{exc}}(f)$ components, is shown to naturally emerge from the statistical features of the domain wall processes [Bertotti 1998]. The STL, with its simple analytical formulations for $W_{\mathrm{exc}}(f)$ and $W_{\mathrm{cl}}(f)$, has found general application in the literature, encompassing different materials (non-oriented and grain-oriented steel sheets, amorphous and nanocrystalline alloys, $\mathrm{Fe}-\mathrm{Co}$ alloys, etc.) and different excitation regimes [Amar 1995, Atallah 1994, Barbisio 2004, Kowal 2015]. It takes at face value the stochastic nature of the domain wall processes, which are assumed to occur, on the average, homogeneously across the sheet crosssection. Under this assumption, the classical loss component can be calculated considering the regular macroscopic eddy current patterns resulting from the long range superposition of the microscopic eddy currents surrounding the moving domain walls. It is therefore obtained, for sinusoidal induction of peak value $B_{\mathrm{p}}$ at frequency $f$ in a sheet of conductivity $\sigma$ and thickness $d$

$$
W_{\mathrm{cl}}(f)=\left(\pi^{2} / 6\right) \cdot \sigma d^{2} B_{\mathrm{p}}^{2} f . \quad\left[\mathrm{J} / \mathrm{m}^{3}\right]
$$

The counterfield generated by the macroscopic eddy currents does actually lead, depending on frequency, to an induction profile across the sheet thickness, but the experiments show that, as far as the flux penetration (skin depth) $\delta$ is larger than the sheet halfthickness, the assumption of uniform induction is tenable and (1) applies whatever the magnetization curve [Beatrice 2014]. In the limiting case of linear material of given permeability $\mu$, the classical loss is straightforwardly obtained from Maxwell's equations as

$$
W_{\mathrm{cl}}(f)=\frac{\pi}{2} \cdot \frac{\gamma B_{\mathrm{p}}^{2}}{\mu} \cdot \frac{\sinh \gamma-\sin \gamma}{\cosh \lambda-\cos \gamma}, \quad\left[\mathrm{J} / \mathrm{m}^{3}\right]
$$


where $\gamma=\sqrt{\pi \sigma \mu d^{2} f}$. This equation is reduced to (1) for $\gamma=d / \delta \leq 2$. It has however been pointed out in recent times [Zirka 2010, Zirka 2015] that the use of (1) would not be a good approximation for $W_{\mathrm{cl}}(f)$ in actual materials excited at technical $B_{\mathrm{p}}$ values, that is, with the magnetization regimes most frequently encountered in applications, because the condition of homogeneous magnetization reversal would not be generally fulfilled. The discrepancy between the actual evolution of the magnetization process and the one assumed by introducing (1) in the loss decomposition by the STL would be especially acute at high inductions, where the material constitutive equation is highly nonlinear, with the quasi-static hysteresis loop in non-oriented (NO) steel sheets taking a near-rectangular shape. Numerical solution of the Maxwell's diffusion equation in combination with a static magnetic hysteresis model in standard NO sheets appears indeed to substantiate the idea that in such a case the magnetization reversal proceeds by propagation of symmetric fronts across the slab [Zirka 2010]. This process would emulate the predicted response of an ideal material with step-like magnetization, where the induction can only attain the values $B= \pm B_{\max }$ for positive and negative fields, respectively [Bertotti, 1998]. If this is the case, the classical loss figures would become, at sufficiently high $B_{\mathrm{p}}$ values,

$W_{\mathrm{cl}}^{(\mathrm{SWM})}(f)=\frac{\pi^{2}}{4} \cdot \sigma d^{2} B_{\max }^{2} f \cdot \frac{B_{\mathrm{p}}}{B_{\max }}=\frac{3}{2} W_{\mathrm{cl}}(f) \cdot \frac{B_{\mathrm{p}}}{B_{\max }},\left[\mathrm{J} / \mathrm{m}^{3}\right]$

where $B_{\max }$ is (somewhat ambiguously) taken as the peak induction associated with a near-rectangular DC loop [Zirka 2010].

Applying (3) to the loss decomposition on typical industrial NO Fe-Si and low-carbon alloys at technical inductions would then likely modify to non-negligible extent the relative roles of classical and excess loss components predicted by the STL using (1), and adjustments would be needed regarding the effects of the structural parameters, flux distortion, sample geometry, etc..

In order to clarify this matter, we have performed and analysed selected measurements on $\mathrm{NO} \mathrm{Fe-Si} \mathrm{and} \mathrm{low-carbon} \mathrm{steel} \mathrm{(LCS)}$ sheets at low and high $B_{\mathrm{p}}$ values. While concluding that the STL using (1) accurately and consistently describe the energy loss versus frequency behaviour in these materials, it is shown that (3) can lead to gross disagreement between predicted and measured losses.

\section{EXPERIMENTAL RESULTS AND DISCUSSION}

Magnetic energy losses have been measured at various peak polarization levels $J_{\mathrm{p}}$ from quasi-static conditions up to maximum frequencies ranging between $400 \mathrm{~Hz}$ and $10 \mathrm{kHz}$ in the $\mathrm{NO} \mathrm{Fe}-\mathrm{Si}$ and low-carbon steel sheets listed in Table 1, tested either as Epstein strips or ring samples by a calibrated wattmeter-hysteresisgraph under digitally controlled sinusoidal induction [Fiorillo 2010]. With their low resistivity value, the LCS are expected to exhibit a relatively high $W_{\mathrm{cl}}(f)$ contribution and favor the appraisal of its role according to either (1) or (3).

Table 1. The investigated non-oriented steel sheets

\begin{tabular}{|c|c|c|c|c|}
\hline Material & Sample & $\begin{array}{c}\text { Thickness } \\
(\mathrm{mm})\end{array}$ & $\begin{array}{c}\text { Density } \\
\left(\mathrm{kg} / \mathrm{m}^{3}\right)\end{array}$ & $\begin{array}{c}\text { Resistivity } \\
(\Omega \mathrm{m})\end{array}$ \\
\hline NO Fe-Si 1 & Ring & 0.194 & 7650 & $52.0 \cdot 10^{-8}$ \\
\hline NO Fe-Si 2 & Epstein & 0.345 & 7600 & $56.0 \cdot 10^{-8}$ \\
\hline
\end{tabular}
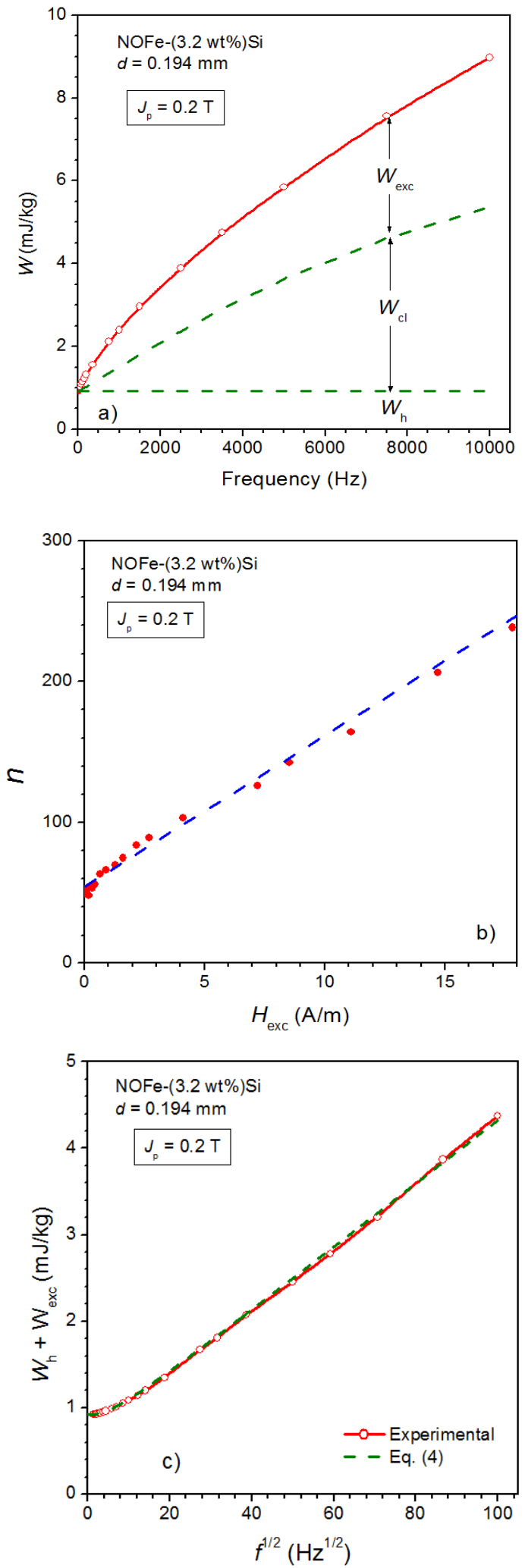

Fig.1 NO Fe-(3.2 wt\%Si) $0.194 \mathrm{~mm}$ thick sheets. a) Decomposition of the measured energy loss (symbols) at $J_{p}=$ $0.2 \mathrm{~T}$ up to $10 \mathrm{kHz}$. The classical loss $W_{\mathrm{cl}}(f)$ is calculated with (2), taking into account the skin effect. b) The number $n$ of active MOs follows a linear dependence $n=n_{0}+H_{\text {exc }} / V_{0}$ on $H_{\text {exc }}=W_{\text {exc }} / 4 J_{\text {p }}$. c) $\left(W_{\mathrm{h}}+W_{\text {exc }}(f)\right)$ versus $f^{1 / 2}$. The experimental excess loss $W_{\text {exc }}(f)$ (symbols) is predicted by (4) (dashed line). 


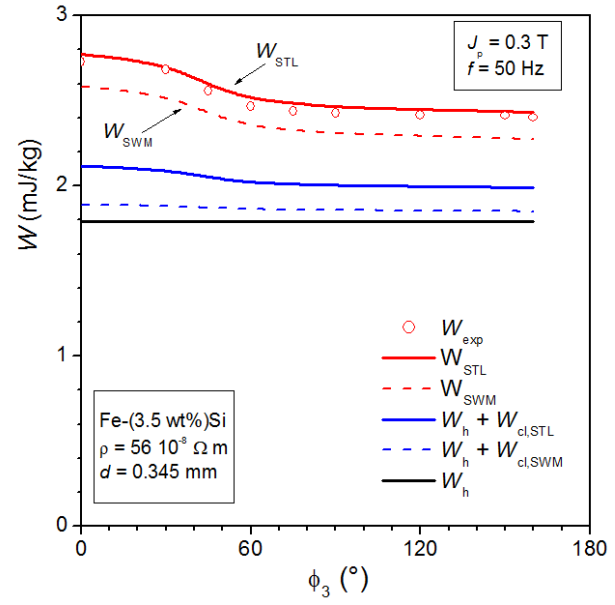

Fig. 2. NO Fe-Si sheet. Measured loss at $50 \mathrm{~Hz}$ and $J_{\mathrm{p}}=$ $0.3 \mathrm{~T}$ under induction distorted by a third harmonic in the ratio $J_{3} / J_{1}=0.2$ and phase $\phi_{3}$ ranging between $0^{\circ}$ and $160^{\circ}$. Symbols: measured loss $W_{\text {exp }}$. Solid lines: prediction by the STL. Dashed lines: prediction by the SWM.

\begin{tabular}{|c|c|c|c|c|}
\hline Low-C steel 1 & Epstein & 0.506 & 7850 & $19.2 \cdot 10^{-8}$ \\
\hline Low-C steel 2 & Ring & 0.507 & 7860 & $15.3 \cdot 10^{-8}$ \\
\hline Low-C steel 3 & Epstein & 0.636 & 7870 & $12.0 \cdot 10^{-8}$ \\
\hline
\end{tabular}

\section{A. Energy losses at low inductions}

Fig. 1 provides full analysis of the loss decomposition according to the STL at $J_{\mathrm{p}}=0.2 \mathrm{~T}$ in a $0.194 \mathrm{~mm}$ thick Fe-(3.2 wt\%)Si sheet up to $f=10 \mathrm{kHz}$. The DC hysteresis loop at his low $J_{\mathrm{p}}$ value is described by the Rayleigh law, where the permeability $\mu=J_{\mathrm{p}} / H_{\mathrm{p}}$ coincides with the differential permeability averaged upon a loop branch between $\pm J_{\mathrm{p}}$ (relative $\mu_{\mathrm{r}}$ at $J_{\mathrm{p}}=0.2 \mathrm{~T}=5900$ ). We can then confidently calculate $W_{\mathrm{cl}}(f)$ using (2) and, once obtained the hysteresis component $\mathrm{W}_{\mathrm{h}}$ by extrapolating $W(f)$ to $f=0$, secure the excess loss $W_{\text {exc }}(f)$, as shown in Fig. 1a. Remarkably, $W_{\text {exc }}(f)$ complies upon the whole investigated frequency range with the theoretically assumed linear dependence $n=n_{0}+H_{\text {exc }} / V_{0}$ of the number $n$ of active magnetic objects (MOs) on the excess field $H_{\text {exc }}$ $=W_{\text {exc }} / 4 J_{\mathrm{p}}$, as illustrated in Fig. $1 \mathrm{~b}$. The quantity $V_{0}$ lumps the effect of the local coercive fields and the experimental $n$ values are obtained as $n=4 \sigma G S J_{\mathrm{p}} / H_{\mathrm{exc}}$, with $G=0.1356$ and $S$ the sample cross-sectional area [Bertotti 1998]. It is shown in Fig. 1c (dashed line) that with the so-obtained $n_{0}$ and $V_{0}$ parameters, the experimental $W_{\text {exc }}(f)$ behavior is excellently described by the theoretical law [Barbisio 2004]

$W_{\mathrm{exc}}(f)=2 n_{\mathrm{o}} V_{\mathrm{o}} J_{\mathrm{p}} \cdot \int_{0}^{\pi / 2}\left(\sqrt{1+\frac{8 \sigma G S V_{\mathrm{o}}}{n_{\mathrm{o}}^{2} V_{\mathrm{o}}^{2}} \pi f J_{\mathrm{p}} \cos \phi}-1\right) \cos \phi \mathrm{d} \phi$.

On the other hand, the notion of saturation wave model (SWM) and the related use of (3) for $W_{\mathrm{cl}}(f)$ is conceptually ambiguous regarding the skin effect at such low inductions and eventually leads to overestimated $W_{\text {exc }}(f)$. This is clear, for example, when predicting the dependence of the energy loss on the distortion of the induction waveform, like in the example provided in Fig. 2, regarding a 0.345 $\mathrm{mm}$ thick NO Fe-(3.5 wt\%)Si. Here total energy loss at $50 \mathrm{~Hz}$ and $J_{\mathrm{p}}$ $=0.3 \mathrm{~T}$ is measured upon the introduction of a third harmonic $J_{3}$

$J(t)=K\left(\phi_{3}\right)\left[\sin (2 \pi f t)+\left(J_{3} / J_{1}\right) \sin \left(6+\phi_{3} \pi f t\right)\right]$,

with $J_{3} / J_{1}=0.2$, the phase shift $\phi_{3}$ varying between $0^{\circ}$ and $160^{\circ}$, and the constant $K(\phi)$ adapted for each $\phi$ so as maintain constant the $J_{\mathrm{p}}$ value (no local minima of $B(t)$ ). According to the STL we write

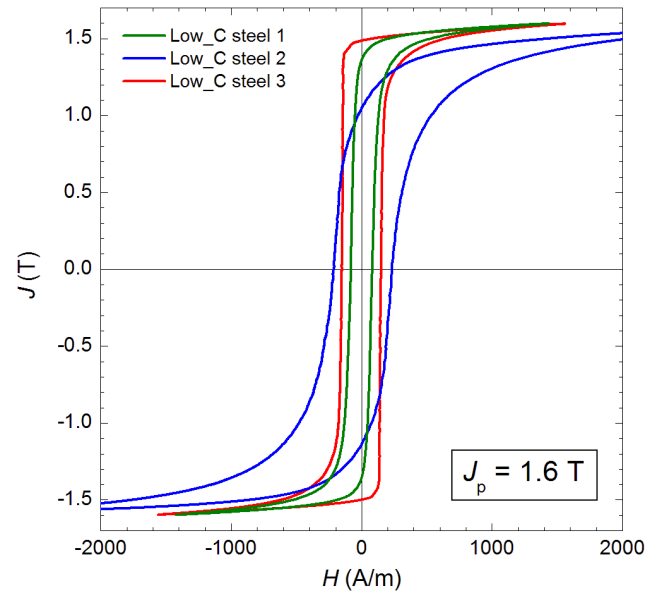

Fig. 3. DC hysteresis loops at $J_{p}=1.6 \mathrm{~T}$ in the three investigated low-carbon steel sheets (see Table 1).

$W_{\mathrm{cl}, \mathrm{STL}}^{\mathrm{dist}}(f)=\frac{1}{12} \sigma d^{2} \int_{0}^{\pi / 2}\left(\frac{d B}{d t}\right)^{2} \mathrm{~d} t \quad$,

while the SWM model would provide [Bertotti 1998]

$W_{\mathrm{cl}, \mathrm{SWM}}^{\text {dist }}=\frac{\sigma d^{2}}{8} \frac{B_{\mathrm{p}}}{B_{\max }} \cdot \int_{0}^{1 / f}\left[\frac{B(t)}{B_{\max }}\left|\frac{d B}{d t}\right| \frac{d B}{d t}+\left(\frac{d B}{d t}\right)^{2}\right] d t$,

with $B_{\max }=1.5 \mathrm{~T}$. The excess loss under the distorted $J(t)$ is given by [Barbisio 2004]

$W_{\mathrm{exc}}=\frac{n_{\mathrm{o}} V_{\mathrm{o}}}{2} \int_{0}^{1 / f}\left(\sqrt{1+\frac{4 \sigma G S_{\mathrm{o}} V_{\mathrm{o}}}{n_{\mathrm{o}}^{2} V_{\mathrm{o}}^{2}}|\dot{J}(t)|}-1\right)|\dot{J}(t)| d t$,

with the parameters $n_{0}$ and $V_{0}$ obtained, as previously discussed, by a measurement at the same $J_{\mathrm{p}}$ value under sinusoidal induction. It is apparent in Fig. 2 that the excellent predictive capability of the STL compares with a too low $W_{\mathrm{cl}}(f)$ prediction by the SWM.

\section{$B$. Energy losses at high inductions}

The step-like $B(H)$ constitutive equation assumed in the SWM can be approximated by the relatively sharp DC hysteresis loops measured on non-oriented steel steels when excited at high $J_{\mathrm{p}}$ values. Fig. 3 shows three such loops, pertaining to the here investigated LCS samples (see Table 1), where we further note that for all practical purposes $B_{\mathrm{p}} \equiv J_{\mathrm{p}}$. Should the SWM and (3) apply, according to the prediction of reversal by propagation of saturation magnetization wavefronts in such strongly non-linear materials [Zirka 2010] [Zirka 2015], some adjustment of the STL would be needed at high inductions. Because of the good conductivity of the LCS, the role of $W_{\mathrm{cl}}(f)$ is emphasized in the present case and application of either (1) or (3) could imply substantial discrepancies on the correspondingly identified excess loss $W_{\text {exc }}(f)$. A sound comparison requires, however, that it be carried out below the frequency range where the skin phenomena enter into play. We cannot obviously make use of (2), as previously done in the Rayleigh region (Fig. 1), in this non-linear context, but the STL provides a unique way to identify incipient skin effect. We follow then the usual procedure, by making the subtraction $W(f)-W_{\mathrm{cl}}(f)$ with the classical loss calculated with (1), that is without considering the skin effect, to obtain $W_{\mathrm{h}}=\lim _{f \rightarrow 0} W(f)$ and $W_{\text {exc }}(f)$. By 
correspondingly finding, as previously discussed, the number $n$ of MOs versus $H_{\text {exc }}(f)=W_{\text {exc }} / 4 J_{\mathrm{p}}$, we realize that, contrary to the lowinduction case shown in Fig. $1 \mathrm{~b}, n\left(H_{\text {exc }}\right)$ does follow the linear relationship $n=n_{0}+H_{\text {exc }} / V_{0}$ only below a certain critical $H_{\text {exc }}$ value. This translates into a divergence between the prediction of $W_{\text {exc }}(f)$ by (4) and its actual behavior beyond a certain limiting frequency, where the skin effect makes (1) inapplicable. The example provided in Fig. 4, concerning the characterization of the LCS 2 sample $(d=$ $0.507 \mathrm{~mm}$ ) at $J_{\mathrm{p}}=1.6 \mathrm{~T}$, shows that in this case one can excellently fit the experimental $W_{\text {exc }}(f)$ with (4) up to about $f=500 \mathrm{~Hz}$, before making room for the skin effect. Using this procedure it is obtained that the skin-effect free region extends up to $f \cong 60 \mathrm{~Hz}$ in LCS 1 and $f \cong 80 \mathrm{~Hz}$ in LCS 3 , both materials displaying much larger permeability than LCS 2 (see Fig. 3).

Let us then consider the loss separation procedure in the three investigated LCS sheets for the highly non-linear regimes at $J_{\mathrm{p}}=1.6$ $\mathrm{T}$. We shall restrain the analysis to the frequency region not affected

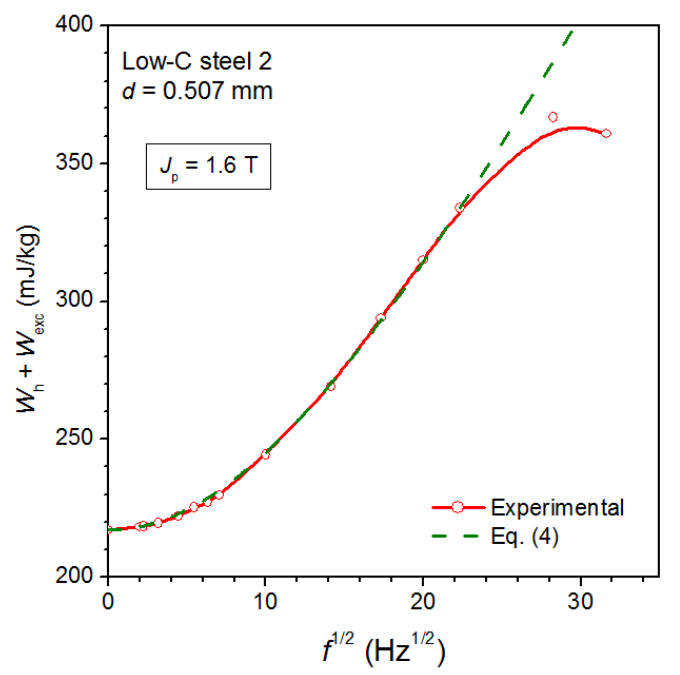

Fig. 4. LCS 2 sheet. Experimental quantity $W_{\mathrm{h}}+W_{\mathrm{exc}}=W-W_{\mathrm{cl}}$, with $W_{\mathrm{cl}}$ given by (1), versus the same quantity predicted with (4) (dashed line). Predicted and experimental curves tend to diverge beyond about $500 \mathrm{~Hz}$, signalling incipient skin effect.

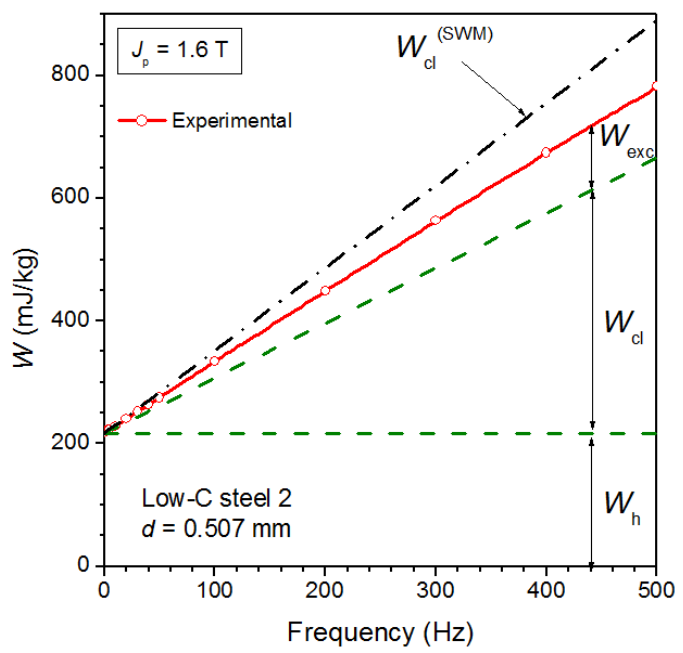

Fig. 5. Decomposition of the energy loss measured at $J_{p}=1.6 \mathrm{~T}$ in the LCS 2 sample upon the skin-effect free frequency region. The classical loss $W_{c l}$ is obtained by (1). The dash-dot line is obtained calculating the classical loss $W_{\mathrm{cl}}$ (SWM) with (3), where it is posed $B_{\max }=B_{\mathrm{p}} . \mathrm{W}_{\mathrm{cl}}{ }^{(\mathrm{SWM})}$ overestimates the measured loss. by the skin effect. The classical loss will be calculated with both (1) and (3), where we shall assume $B_{\max } \equiv B_{\mathrm{p}} \equiv J_{\mathrm{p}}=1.6 \mathrm{~T}$. In such a case we have $W_{\mathrm{cl}}^{(\mathrm{SWM})}(f)=\frac{3}{2} W_{\mathrm{cl}}(f)$ and for the LCS 2 sample we obtain, as shown in Fig. 5, that $W_{\mathrm{cl}}^{(\mathrm{SWM})}(f)$ overestimates the experimentally measured loss, besides leaving no room for the excess losses. This contrasts with the idea that the very existence of moving domain walls and of the related localized eddy currents should in any case provide an extra-loss contribution, whatever assumption is made regarding the uniformity of the distribution of the flux density at low frequencies [Mayergoyz 1999]. The experiments further show that (3) equally overestimates the loss measured at $J_{\mathrm{p}}=1.5 \mathrm{~T}$, although to a lower extent with respect to $J_{\mathrm{p}}$ $=B_{\max }=1.6 \mathrm{~T}(7 \%$ versus $10 \%$ at $f=300 \mathrm{~Hz})$. This occurs in spite of the idea that the higher is $J_{\mathrm{p}}$ the closer is the magnetization curve to a step-like function. Figs. 6 and 7, concerning the LCS 1 and LCS 3 sheet samples, equally demonstrate the inadequacy of (3) in assessing the measured loss versus frequency behaviour, although upon a more restricted frequency range, as imposed by earlier surge of the skin effect.

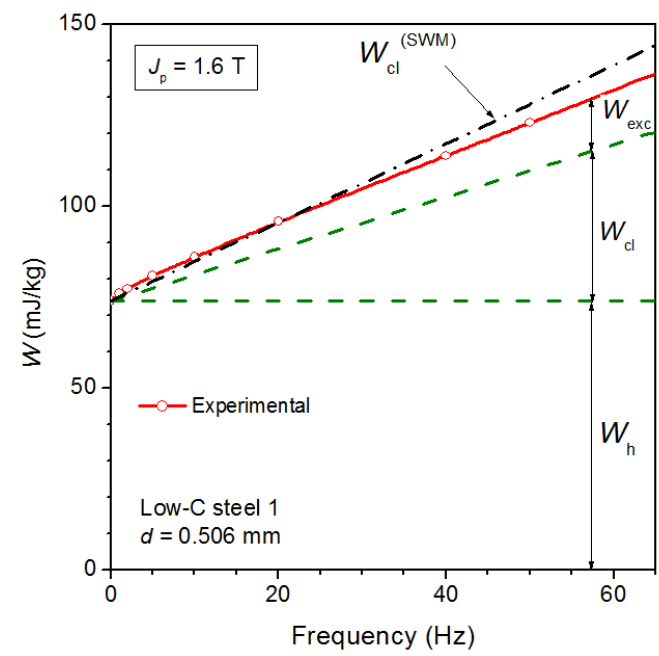

Fig. 6. As in Fig. 5 for the LCS 1 sheet sample.

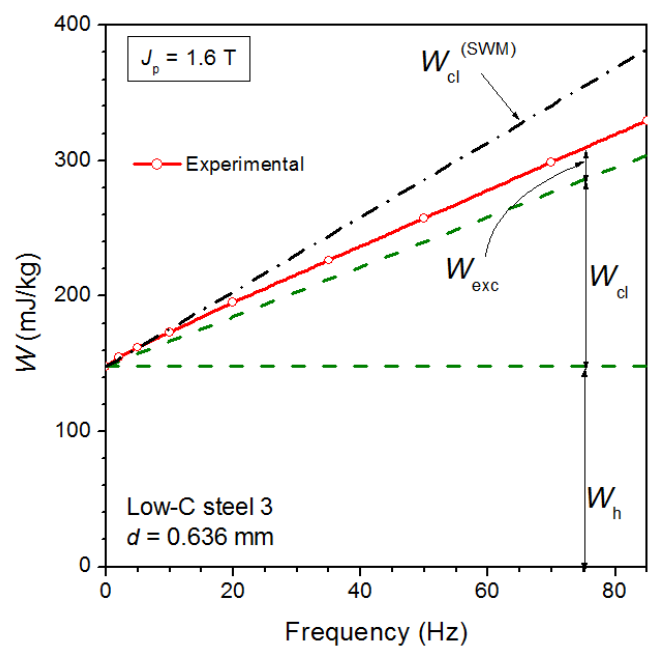

Fig. 7. As in Fig. 5 for the LCS 3 sheet sample. 


\section{CONCLUSION}

It is shown by experiments on different types of non-oriented steel sheets that the magnetic energy losses can be consistently and accurately assessed at low and high inductions using the concept of loss decomposition, in the framework of the Statistical Theory of Losses. This is, in particular, achieved, within the constraints posed by the skin effect, assuming uniform reversal of the magnetization across the sheet cross-section at the macroscopic scale and corresponding standard formulation for the classical loss component. By making measurements at $J_{\mathrm{p}}=1.6 \mathrm{~T}$ in low-carbon steels, one can put in evidence that the previous assumption still holds, although the $B(H)$ constitutive equation tends to emulate a step-like function. This would theoretically imply the occurrence of the magnetization reversal by inward motion of one-dimensional fronts. The ensuing formulation for the classical loss turns however to grossly overestimate the measured losses and can be ruled out.

\section{REFERENCES}

Amar M, Kaczmarek R (1995), "A general formula for prediction of iron losses under nonsinusoidal voltage waveform," IEEE Trans. Magn., vol. 31, pp. 2504-2509, doi: $10.1109 / 20.406552$.

Atallah K, Howe D (1994), "The calculation of iron losses in brushless permanent magnet DC motors," J. Magn. Magn. Mater., vol. 133, pp. 578-582, doi:10.1016/0304-8853(94)90627-0

Barbisio E, Fiorillo F, Ragusa C (2004), “ Predicting loss in magnetic steels under arbitrary induction waveform and with minor hysteresis loops," IEEE Trans. Magn., vol. 40, pp. 1810-1819, doi: 10.1109/TMAG.2004.830510.

Beatrice C, Appino C, de la Barrière O, Fiorillo F, Ragusa C (2014), "Broadband magnetic losses in Fe-Si and Fe-Co laminations," IEEE Trans. Magn.., vol. 50, pp. 6300504, doi: 10.1109/TMAG.2013.2286923

Bertotti G (1998), Hysteresis in Magnetism, San Diego, CA: Academic Press, 1998, pp. $391-430$.

Fiorillo F (2010), "Measurements of magnetic materials," Metrologia, vol. 47, pp. S114S142, doi:10.1088/0026-1394/47/2/S11.

Kowal D, Sergeant P, Dupré L, Vandenbossche L (2015), “Comparison of iron loss models for electrical machines with different frequency domain and time domain methods for excess loss prediction," IEEE Trans. Magn., vol. 51, pp. 6300110, doi:10.1109/TMAG.2014.2338836.

Mayergoyz I, Serpico C (1999), "Nonlinear diffusion of electromagnetic fields and excess eddy current losses," J. Appl. Phys., vol 85, pp. 4910-4912, doi: 10.1063/1.369139

Zirka S E, Moroz Y I, Marketos P, Moses A J (2010), "Loss separation in nonoriented electrical steelsComparison of iron loss models for electrical machines with different frequency domain and time domain methods for excess loss prediction," IEEE Trans. Magn., vol. 51, pp. 6300110, doi:10.1109/TMAG.2014.2338836.

Zirka S E, Moroz Y I, Steentjes S, Hameyer K, Chwastek K, Zurek S, Harrison R G (2015), "Dnamic magnetization models for soft ferromagnetic materials with coarse and fine domain structures," J. Magn. Magn. Mater., vol. 394, pp. 229-236, doi:10.1016/j.jmmm.2015.06.082. 